\title{
324 - Twelve-month progress of online intervention to prevent cognitive decline: Maintain Your Brain.
}

Authors: Henry Brodaty, Megan Heffernan, Gavin Andrews, Kaarin Anstey, Maria Fiatarone Singh, Louisa Jorm, Nicola Lautenschlager, Anthony Maeder, John McNeill, Perminder Sachdev, Michael Valenzuela, Maintain Your Brain Collaborative Team.

Abstract: In the absence of disease modifying interventions for Alzheimer's disease (AD) and other dementias there is an increased interest in dementia prevention. An issue for population-based lifestyle preventative approaches is scalability. An internet-based multicomponent Maintain Your Brain (MYB) randomised trial is currently underway.

Invitations to participate in Maintain Your Brain were emailed or mailed to people aged 55-77 years from the 45 and Up study, a population-based cohort study of one in ten people aged 45 years and older $(n=267,000)$. To be enrolled into the study participants were required to have risk factors that made them eligible for at least one of the available modules. Modules were designed to address physical inactivity and health risks associated with inactivity (Physical Activity), adherence to a Mediterranean-type diet and health risks associated with poor nutrition (Nutrition), cognitive activity (Brain Training) and mental wellbeing (Peace of Mind).

During recruitment 96,418 invitations were sent and 14,064 (14\%) provided consent. Of those who completed baseline over $90 \%$ were eligible for at least two modules of the intervention. Overall, $6,236(44 \%)$ were enrolled resulting in an overall recruitment rate of $6 \%$, or $50 \%$ of those eligible at screening. The final sample was $64 \%$ female with a mean age of $64.9 \mathrm{yrs}$ ( $S D=5.8$, range $55-77 \mathrm{yrs}$ ) and mean years of education of $12.9 \mathrm{yrs}(\mathrm{SD}=3.0$, range $2-22 \mathrm{yrs}$ ).

Recruitment rates in MYB were comparable to other clinical trials targeting older people and who included online recruitment strategies. This is promising for the willingness of participants to engage with trials that use online rather than traditional in-person methods. Although the baseline withdrawal number (3\%) remained stable after randomisation (4\%), a further $25 \%$ of participants did not attempt any activities. These rates continued at the annual assessments when $4 \%$ withdrew, $31 \%$ completed no follow-up activities, $14 \%$ partially completed and $55 \%$ completed all.

Online lifestyle interventions have capacity to reach broad segments of the 55-77 year old population, the majority of whom may benefit from support activities shown to be effective in reducing risk of cognitive decline and ultimately delay onset of dementia. The challenge with this new approach is encouraging continued engagement with the program over time. 